\title{
Human SLC11A1 gene polymorphism has the propensity to confer susceptibility to M. africanum TB disease in Ghana
}

4 Adwoa Asante-Poku ${ }^{1,2,3 *}$, Portia Morgan ${ }^{1,2,3}$, Samuel Yaw Aboagye ${ }^{1}$, Prince Asare ${ }^{1,2,3}$, Isaac Darko Otchere ${ }^{1,2}$, Samuel Mawuli

5 Adadey $^{5}$, Khuthala Mnika ${ }^{5}$, Kenneth Hayibor Mawuta ${ }^{1,2}$, Nelly Arthur ${ }^{4}$, Audrey Forson ${ }^{4}$, Gaston Kuzamunu ${ }^{5}$, Ambroise Wonkam ${ }^{5}$,

6 Dorothy Yeboah-Manu ${ }^{1,2,3}$

\section{Affiliations} poku@noguchi.ug.edu.gh(A.A.P);portia.morgan33@gmail.com(P.M); Research, University of Ghana; aasanteasare.prince@hotmail.com (P.A); idotchere@gmail.com (I.D.O); kenhayibor@gmail.com (K.H.M)

2 West African Centre for Cell Biology of Infectious Pathogens, University of Ghana; aasante-poku@noguchi.ug.edu.gh(A.A.P); portia.morgan33@gmail.com(P.M); asare.prince@hotmail.com(P.A); idotchere@gmail.com (I.D.O); kenhayibor@gmail.com (K.H.M); dyeboah-manu@noguchi.ug.edu.gh (D.Y.M)

3 Department of Biochemistry, Cell and Molecular Biology, University of Ghana; aasante-poku@noguchi.ug.edu.gh(A.A.P); portia.morgan33@gmail.com (P.M); asare.prince@hotmail.com (P.A); $\underline{\text { idotchere@gmail.com }}$ (I.D.O); dyeboahmanu@noguchi.ug.edu.gh (D.Y.M)

4 Department of Chest Diseases, Korle-Bu Teaching Hospital, Korle-Bu, Accra, Ghana; nellyarthur6776@gmail.com (N.A); agforson@chs.edu.gh (A.F) 
21

22

23

24

25 Corresponding Author

26 Name: Adwoa Asante-Poku

27 Address: Noguchi Memorial Institute for Medical Research, P. O. Box LG 581, Accra-Ghana

28 Email: aasante-poku@noguchi.ug.edu.gh

29

30 Key Words

31 Tuberculosis; Host pathogen interactions; SNPs

32

33

34

35

36

37

38

39

40

41 mnikakhuthala@gmail.com (K.M); gmazandu@gmail.com (G.M); ambroise.wonkam@uct.ac.za (A.W)

3

2

33

35

6

38

9

0

1

5 Division of Human Genetics, Faculty of Health Sciences - University of Cape Town; adadeymawuli.am@gmail.com; (S.M.A) 
42 Abstract:

43 Human tuberculosis (TB) is caused mainly by Mycobacterium tuberculosis (MTB) and M. africanum (MAF) remains a major global 44 health threat. The varying response of different host to contact with the TB bacteria, indicates the importance of host genetics in 45 susceptibility to TB disease. We explored the association among selected human/host genomic variants and disease caused by the two 46 causative pathogens in Ghana through a case control study. MTBC isolates (323) recovered from pulmonary TB patients recruited 47 between 2016 and 2018 were genotyped using spoligotyping. A selection of 29 SNPs from MTB-related genes with high frequency 48 among African populations were genotyped using a TaqMan® SNP Genotyping Assay and iPLEX Gold Sequenom Mass Genotyping 49 Array. Associations between MTBC lineages and host variables were assessed using univariate and multivariate logistic regression.

50 The prevalence of MTB and Maf among the participants were 79\% and 21\% respectively. Association analysis between the controls 51 and MAF showed that rs2695342 variant on the SIC11A1 gene have the propensity to confer susceptibility to MAF infections $(P=$ $520.093, \mathrm{OR}=8.35,95 \% \mathrm{CI}=0.70-99.24)$ whilst the $\mathrm{rs} 17048476(P=0.088, \mathrm{OR}=1.57,95 \% \mathrm{CI}=0.93-2.63)$ and $\mathrm{rs} 1482868(P=$ $530.095, \mathrm{OR}=0.60,95 \% \mathrm{CI}=0.33-1.09)$ were observed to be only suggestive. Our findings implicate SLC11A1 as a potential 54 susceptibility gene of substantial interest for TB caused by MAF which is an important pathogen in West Africa and highlight the 55 need for in-depth host pathogen studies in West Africa.

56

57

58

Keywords: Tuberculosis, M. africanum, SLC11A1

59

60

61

62 
66

68

69

\section{INTRODUCTION}

Pulmonary tuberculosis (TB), is a significant public health burden worldwide, with 10 million cases and an estimated 1.5 million deaths in 2018(1). As a disease transmitted by the inhalation of aerosolized droplets, [1], TB is expected that susceptible individuals who come into contact with aerosol(s) containing viable bacteria will be equally infected. In about $90 \%$ of infected individuals, between 3 to 8 weeks after MTB contained in inhaled aerosols becomes implanted in alveoli (1), the host immune system comprising of both the innate and adaptive arm will wall off the site if infection in a granuloma (ghon complex), such individuals are asymptomatic and are latent TB infection (LTBI) [2-4]. Only 5\% of infected immune competent individuals will potentially develop active TB disease within 2-3 years of infection while the remaining 5\% develop TB later in their life [5-8]. This difference potentially depends on the interplay between the environment, the bacteria and most importantly the host genetic factors associated with TB pathogenesis.

For many years, the diverse impact of several important genes such as the major histocompatibility complex (MHC), and non-HLA genes like killer immunoglobulin-like receptor (KIR), toll-like receptors (TLRs), cytokine/chemokines and their receptors, vitamin D receptor (VDR), SLC11A1 and C-type lectins on the susceptibility to TB or otherwise has been demonstrated by several studies (8-10). For instance, In 2012, Pydi et al reported Inhibitory genes KIR3DL1, KIR2DL3 conferring susceptibility towards TB in individuals and recently, Bartlelt et in 2020 observed significant interaction between a single nucleotide polymorphism (SNP) in SLC11A1 and the L4-Ugandan lineage in TB household contact study in Kampala, Uganda [9-10]. Indeed, familial risk of tuberculosis has been recognized for centuries, largely through studies of mono- and dizygotic twin concordance rates, studies of families with Mendelian susceptibility to mycobacterial disease, and candidate gene studies performed in the 20th century leading to the realization that susceptibility to TB disease has a substantial host genetic component [2-8]. 
82 Although anatomical modern humans evolved from Africa, very few genetic studies have been conducted in ancestral Africans with

83 regards to TB host genetic susceptibility. Therefore, our knowledge of even the most fundamental information on the genetic basis

84 of susceptibility or otherwise to TB disease in Africa is quite limited. Moreover, only few studies have taken into consideration the

85 potential influence of different genotypes of the TB pathogen on such interactions. Human TB is mainly caused by Mycobacterium

86 africanum (MAF) and M. tuberculosis sensu stricto (MTB), both members of $M$. tuberculosis complex (MTBC), which also includes

87 several sub-species adapted to a variety of wild and domestic animals [2-3]. Of special interest to Africa is MAF, which is restricted to

88 West Africa and may cause up to $50 \%$ of human TB in some of the countries unlike the globally ubiquitous MTB. In Ghana for

89 instance, MAF causes about $20 \%$ of all TB cases [2]. Thus, host genetics and susceptibility to distinct MTBC lineages cannot be

90 overlooked. Findings from two independent molecular epidemiological studies by our group showed a strong association between

91 MAF (driven by lineage 5) and a native West African ethnic group [11-12]. This current study thence explored potential host and

92 pathogen interactions towards understanding MAF and MTB infections to enhance our understanding of TB pathogenesis

93

94

95

96

97

98

99

100

101 


\section{MATERIALS AND METHODS}

\section{2.1. Ethical Approval}

104 The study was performed in accordance with the Declaration of Helsinki. Ethical approval for the study was obtained from the

105 Noguchi Memorial Institute for Medical Research Institutional Review Board (NMIMR-IRB 097/15-16). Written and signed informed

106 consent was obtained from participants before enrolment into the study

\section{$107 \quad$ 2.2. Study population}

108 Our study population were in two categories: Diagnosed TB patients' group and non-TB patients serving as control group (NTB). For

109 the TB group, only newly diagnosed sputum smear-positive adult TB cases registered at the Department of chest disease, Korle-bu

110 teaching Hospital in Ghana were recruited into the study before the commencement of TB treatment from $1^{\text {st }}$ July $2016-31^{\text {st }}$ July

111 2018. All patients were unrelated. Ethnicity was defined in line with Ghana demographic data 2010 [13].

112 For the NTB group, 5 communities' sites by the National TB control programme were selected. These sites included Korle-gonno

113 (Ablekuma south sub metro), Bukom (Ashiedu Keteke sub metro), Abossey-okai (Okaikoi south), Glefe (Ablekuma West) and

114 Amanfrom (Ga West District). Medical outreaches were conducted in these sites and with a new structured questionnaire, clinical

115 characteristics (diabetes, HIV, hypertension) as well demography and epidemiological data were obtained from each participant.

116 Only patients with no history of TB were recruited into the study. In accordance with ethics, participants with elevated stage 1 or 2

117 hypertension were transported to the nearest hospital for immediate medical attention and were excluded from the study.

\section{2.2. Sample collection}

120 Diagnosed TB patients' group 
121 To confirm the initial diagnosis at the health facility and to identify the infecting mycobacterial species, sputum specimen was

122 collected from each TB study participant, following the National Tuberculosis Control Program guidelines. Samples were taken only

123 after a detailed explanation of the study and written, or thumb-printed consent have been obtained for participation. Clinical

124 characteristics (previous history of TB, HIV) as well demography and epidemiological data including age, sex and ethnic origin were

125 obtained from each participant. In addition, each patient was screened for Diabetes mellitus (DM) using random blood glucose

126 level. Based on the American Diabetes Association (ADA) criteria, a glucometer (ACCU-CHEK) Active Glucose Monitoring System,

127 Roche Diabetes Care Limited, Burgess Hill, UK which uses finger prick test OneTouch Ultra test strips was used to screen all patients

128 for DM irrespective of known DM status. If the blood glucose level was less than 7mmol/L, no further action was taken, however if

129 blood glucose level was above $13 \mathrm{mmol} / \mathrm{L}$, test was confirmed as having Diabetes. $5 \mathrm{ml}$ of blood was collected from each TB patient

130 for host genetics analysis.

131 Non-TB patients control group (NTB) and chest X-ray screening.

132 The screening of eligible control individuals was done in a stepwise manner. Firstly, Adults (>18 years) presenting at any of the 133 outreach centers were verbally screened for fever, diabetes and hypertension. Individuals with no evidence of fever, diabetes or 134 elevated hypertension were then taken through chest X-ray (CXR) screening using CAD4TB (version 3.07, Diagnostic Image Analysis

135 Group, The Netherlands) for abnormalities suggestive of pulmonary TB. The software has two abnormality detection systems that is 136 textural abnormality and shape abnormality systems, which analyze the abnormalities in the unobscured lung fields that have been 137 segmented automatically [14]. A higher score is suggestive of TB. A CAD4TB threshold score of 60 was used for this population 138 determined using previously collected CXR data in a similar population. Sputum samples was collected from all individuals with high 139 CAD4TB scores (60 or greater) and transported to the laboratory for further analysis. Individuals with high CAD4TB scores were 140 referred to the hospitals for further clinical evaluation. $5 \mathrm{ml}$ of blood was collected from each TB patient for host genetics analysis. 


\section{$141 \quad$ 2.3. Laboratory analysis}

142 Isolation and characterization of Mycobacterium spp.

143 Sputum samples obtained were decontaminated using 5\% oxalic acid [15-16] and inoculated on two pairs of Lowenstein Jensen (L)

144 slants; one supplemented with $0.4 \%$ sodium pyruvate to enhance the isolation of MAF and M. bovis, and the other with glycerol for

145 the growth of MTB. The cultures were incubated at $37^{\circ} \mathrm{C}$ and were observed weekly for growth for a maximal duration of 16 weeks.

146 MTBC strains were identified by PCR detection of insertion sequence IS6110 as previously described [16]. Colonies from positive

147 cultures were purified and stored at $-80{ }^{\circ} \mathrm{C}$ in $2 \mathrm{ml}$ Middlebrook $7 \mathrm{H} 9$ supplemented with ADC enrichment media until use. Pure

148 Bacteria DNA was extracted for genotyping using a modified protocol [17] and stored at $-20^{\circ} \mathrm{C}$ until further use.

149 All MTBC isolates were further typed by spoligotyping [18]. This was performed according to the manufacturer's instructions, using

150 commercially available kits (Isogen Bioscience BV Maarssen, The Netherlands). Briefly, The DR containing region was amplified by

151 PCR using primers, DRa and DRb (GGTTTTGGGTCTGACGAC, and CCGAGGGGACGGAAAC). The amplified products were hybridized to

152 set of 43 oligonucleotides each corresponding to one spacer, immobilized on a nylon membrane. Detection of hybridization was

153 achieved using chemiluminescent ECL (Amersham) liquid followed by X-ray exposure. The Spoligotyping patterns obtained were

154 defined according to SITVITWEB database (http://www.pasteur-guadeloupe.fr:8081/SITVIT ONLINE). SITVITWEB assigned shared

155 types numbers were used whenever a spoligotyping pattern was found in the database while families and subfamilies were assigned

156 based on the MIRU-VNTRplus database (http://www.miru-vntrplus) (http://www. miru-vntrplus.org). Shared types were defined as

157 patterns common to at least two or more isolates. All patterns that could not be assigned were considered orphan spoligotypes.

158

159

160 


\section{Host DNA Isolation}

162 For all enrolled participants $5 \mathrm{ml}$ of whole blood was drawn into EDTA-coated tubes (BD Biosciences) and immediately stored at $4{ }^{\circ} \mathrm{C}$.

163 DNA was extracted within 24 hours from peripheral blood, following instructions on the available commercial kit Gentra Puregene

164 Blood Kit (QIAGEN) in accordance with the manufacturer's recommendations. All DNA samples were stored at $80{ }^{\circ} \mathrm{C}$ prior to 165 genotyping.

166 DNA quality was evaluated according to the 260/280 ratio with a NanoDrop 2000 spectrophotometer (Thermo Scientific). In total

167792 samples with concentrations of $5 \mathrm{ug} / \mathrm{ul}-851.87 \mathrm{ug} / \mathrm{ul}$ were selected and sent to the whilst single nucleotide polymorphisms 168 (SNPs) typing.

169

170 Host genetics analysis: Genotyping of targeted SNPs in MTB-related host genes

171 Targeted sequencing was conducted in the Division of Human Genetics Laboratory, Faculty of Health Sciences, at the University of 172 Cape Town, South Africa. Genetic variants that are potentially associated with MTB were identified from the recent literature. Once 173 the SNPs of interests were identified, we investigated their allele frequencies in African populations present in the 1000 Genomes 174 project (http://www.internationalgenome.org/home), and further narrowed the selection to SNPs that showed high frequency 175 among African populations. This resulted in the selection of 29 SNPs from MTB-related genes that were investigated in the present 176 study (Table 1). SNPs (Table 1) were genotyped using a TaqMan ${ }^{\circledR}$ SNP Genotyping Assay and TaqMan ${ }^{\circledR}$ Universal Master Mix (Life 177 Technologies, Carlsbad, CA, USA), at the Division of Human Genetics, Faculty of Health Sciences, University of Cape Town; and by 178 iPLEX Gold Sequenom Mass Genotyping Array (Inqaba Biotec, Pretoria, South Africa). Validation was done in a subset of samples 179 (10\%), by Sanger sequencing using BigDye terminator mix (Promega, Madison, WI, USA) (Supplementary figure 1).

180

181 
182
183
184
185
186
187
188
189

182
183
184
185
186
187
188
189

182
183
184
185
186
187
188
189

182
183
184
185
186
187
188
189

182
183
184
185
186
187
188
189

182
183
184
185
186
187
188
189

182
183
184
185
186
187
188
189

182
183
184
185
186
187
188
189

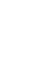


190 Table 1: Allele frequencies of SNPs used in the study

\begin{tabular}{|c|c|c|c|c|c|c|c|c|c|c|}
\hline \multirow[b]{2}{*}{ Gene } & \multirow[b]{2}{*}{ rs number } & \multirow[b]{2}{*}{ Allele } & \multicolumn{2}{|c|}{ Current study } & \multicolumn{6}{|c|}{ Ensembl } \\
\hline & & & Frequency & Ratio & All & AFR & AMR & EAS & EUR & YRI \\
\hline \multirow{2}{*}{ CALN1 } & \multirow{2}{*}{ rs844669 } & A & 593 & 0,74 & 0,68 & 0,72 & 0,57 & 0,75 & 0,61 & 0,74 \\
\hline & & C & 206 & 0,26 & 0,32 & 0,28 & 0,43 & 0,25 & 0,39 & 0,26 \\
\hline \multirow{2}{*}{$S L C 11 A 1$} & \multirow{2}{*}{ rs17235409 } & A & 146 & 0,18 & 0,07 & 0,06 & 0,08 & 0,14 & 0,01 & 0,06 \\
\hline & & G & 653 & 0,82 & 0,93 & 0,94 & 0,92 & 0,86 & 0,99 & 0,94 \\
\hline \multirow{2}{*}{ LOC107984706 } & \multirow{2}{*}{ rs857063 } & A & 599,5 & 0,75 & 0,66 & 0,67 & 0,57 & 0,47 & 0,82 & 0,66 \\
\hline & & C & 199,5 & 0,25 & 0,34 & 0,33 & 0,43 & 0,53 & 0,18 & 0,34 \\
\hline \multirow{2}{*}{$F 13 A 1$} & \multirow{2}{*}{ rs1482868 } & C & 664,5 & 0,83 & 0,69 & 0,75 & 0,74 & 0,76 & 0,66 & 0,75 \\
\hline & & $\mathrm{T}$ & 134,5 & 0,17 & 0,31 & 0,25 & 0,26 & 0,24 & 0,34 & 0,25 \\
\hline \multirow{2}{*}{ PLCD4 } & \multirow{2}{*}{ rs3731869 } & G & 553 & 0,69 & 0,08 & 0,12 & 0,02 & 0,12 & 0,03 & 0,14 \\
\hline & & $\mathrm{T}$ & 246 & 0,31 & 0,92 & 0,88 & 0,98 & 0,88 & 0,97 & 0,86 \\
\hline \multirow{2}{*}{ MBL2 } & \multirow{2}{*}{ rs5030737 } & A & 43,5 & 0,05 & 0,03 & 0 & 0,03 & 0 & 0,06 & 0 \\
\hline & & G & 755,5 & 0,95 & 0,97 & 100 & 0,97 & 100 & 0,94 & 100 \\
\hline \multirow{2}{*}{ ZPLD1b } & \multirow{2}{*}{ rs12636260 } & G & 295 & 0,73 & 0,54 & 0,73 & 0,53 & 0,54 & 0,45 & 0,76 \\
\hline & & $\mathrm{T}$ & 107 & 0,27 & 0,46 & 0,27 & 0,47 & 0,46 & 0,55 & 0,24 \\
\hline \multirow{2}{*}{$F 13 A 1$} & \multirow{2}{*}{ rs1482868 } & C & 277,5 & 0,70 & 0,69 & 0,75 & 0,74 & 0,76 & 0,66 & 0,52 \\
\hline & & $\mathrm{T}$ & 119,5 & 0,30 & 0,31 & 0,25 & 0,26 & 0,24 & 0,34 & 0,48 \\
\hline \multirow{2}{*}{$D A B 1$} & \multirow{2}{*}{ rs1524713 } & $\mathrm{C}$ & 295,5 & 0,72 & 0,69 & 0,75 & 0,74 & 0,76 & 0,66 & 0,75 \\
\hline & & $\mathrm{T}$ & 115,5 & 0,28 & 0,31 & 0,25 & 0,26 & 0,24 & 0,34 & 0,25 \\
\hline \multirow{2}{*}{ Bsm-I } & \multirow{2}{*}{ rs1544410 } & C & 312,5 & 0,74 & 0,7 & 0,73 & 0,74 & 0,94 & 0,6 & 0,7 \\
\hline & & $\mathrm{T}$ & 108,5 & 0,26 & 0,3 & 0,27 & 0,26 & 0,06 & 0,4 & 0,3 \\
\hline GLO1 & rs1616723 & C & 54,5 & 0,17 & 0,12 & 0,18 & 0,05 & 0,18 & 0,08 & 0,19 \\
\hline
\end{tabular}




\begin{tabular}{|c|c|c|c|c|c|c|c|c|c|c|}
\hline & & $T$ & 271,5 & 0,83 & 0,88 & 0,82 & 0,95 & 0,82 & 0,92 & 0,81 \\
\hline \multirow{2}{*}{$A K 124857$} & \multirow{2}{*}{ rs17048476 } & A & 165 & 0,40 & 0,24 & 0,4 & 0,18 & 0,08 & 0,21 & 0,37 \\
\hline & & G & 245 & 0,60 & 0,76 & 0,6 & 0,82 & 0,92 & 0,79 & 0,63 \\
\hline \multirow{2}{*}{ SLC11A1 } & \multirow{2}{*}{ rs17235409 } & A & 23 & 0,05 & 0,07 & 0,06 & 0,08 & 0,14 & 0,01 & 0,06 \\
\hline & & G & 408 & 0,95 & 0,93 & 0,94 & 0,92 & 0,86 & 0,99 & 0,94 \\
\hline \multirow{2}{*}{ RBFOX1 } & \multirow{2}{*}{ rs2346943 } & G & 88,5 & 0,21 & 0,33 & 0,3 & 0,38 & 0,26 & 0,42 & 0,29 \\
\hline & & T & 331,5 & 0,79 & 0,67 & 0,7 & 0,62 & 0,74 & 0,58 & 0,71 \\
\hline \multirow{2}{*}{ ADAMTS14 } & \multirow{2}{*}{ rs2587469 } & A & 225,5 & 0,58 & 0,62 & 0,56 & 0,5 & 0,7 & 0,62 & 0,6 \\
\hline & & G & 165,5 & 0,42 & 0,38 & 0,44 & 0,5 & 0,3 & 0,38 & 0,4 \\
\hline \multirow{2}{*}{ THSD7A } & \multirow{2}{*}{ rs2681052 } & A & 155 & 0,38 & 0,5 & 0,38 & 0,64 & 0,41 & 0,64 & 0,39 \\
\hline & & c & 248 & 0,62 & 0,5 & 0,62 & 0,36 & 0,59 & 0,36 & 0,61 \\
\hline \multirow{4}{*}{ SLC11A1 } & \multirow{2}{*}{ rs2695342 } & G & 422 & 0,97 & 0,98 & 0,94 & 100 & 100 & 100 & 0,96 \\
\hline & & A & 12 & 0,03 & 0,02 & 0,06 & 0 & 0 & 0 & 0,04 \\
\hline & \multirow{2}{*}{ rs2807348 } & A & 330 & 0,78 & 0,45 & 0,72 & 0,41 & 0,33 & 0,26 & 0,77 \\
\hline & & G & 92 & 0,22 & 0,55 & 0,28 & 0,59 & 0,67 & 0,74 & 0,23 \\
\hline \multirow{2}{*}{ RGS3 } & \multirow{2}{*}{ rs3860173 } & $c$ & 234 & 0,57 & 0,63 & 0,65 & 0,62 & 0,39 & 0,75 & 0,59 \\
\hline & & $T$ & 177 & 0,43 & 0,37 & 0,35 & 0,38 & 0,61 & 0,25 & 0,41 \\
\hline \multirow{2}{*}{ SFRP1 } & \multirow{2}{*}{ rs4236914 } & c & 282,5 & 0,72 & 0,79 & 0,73 & 0,79 & 0,72 & 0,95 & 0,68 \\
\hline & & T & 111,5 & 0,28 & 0,21 & 0,27 & 0,21 & 0,28 & 0,05 & 0,32 \\
\hline \multirow{2}{*}{ SLC38A4 } & \multirow{2}{*}{ rs4768760 } & A & 243,5 & 0,58 & 0,61 & 0,59 & 0,61 & 0,61 & 0,66 & 0,59 \\
\hline & & $c$ & 173,5 & 0,42 & 0,39 & 0,41 & 0,39 & 0,39 & 0,34 & 0,41 \\
\hline \multirow{2}{*}{ ADGRL3 } & \multirow{2}{*}{ rs4860106 } & A & 102 & 0,40 & 0,49 & 0,63 & 0,61 & 0,21 & 0,59 & 0,63 \\
\hline & & G & 156 & 0,60 & 0,51 & 0,37 & 0,39 & 0,79 & 0,41 & 0,37 \\
\hline
\end{tabular}


191

\begin{tabular}{|c|c|c|c|c|c|c|c|c|c|c|}
\hline \multirow{2}{*}{ UBLCPI, IL12B } & \multirow{2}{*}{ rs4921437 } & C & 282,5 & 0,68 & 0,86 & 0,74 & 0,89 & 100 & 0,83 & 0,76 \\
\hline & & $\mathrm{T}$ & 130,5 & 0,32 & 0,14 & 0,26 & 0,11 & 0 & 0,17 & 0,24 \\
\hline \multirow{2}{*}{ WNT4 } & \multirow{2}{*}{ rs557438 } & A & 117 & 0,30 & 0,23 & 0,33 & 0,12 & 0,15 & 0,23 & 0,29 \\
\hline & & G & 279 & 0,70 & 0,77 & 0,67 & 0,88 & 0,85 & 0,77 & 0,71 \\
\hline \multirow{2}{*}{ GRIN2B } & \multirow{2}{*}{ rs7297313 } & A & 250,5 & 0,64 & 0,78 & 0,64 & 0,87 & 0,8 & 0,86 & 0,61 \\
\hline & & c & 142,5 & 0,36 & 0,22 & 0,36 & 0,13 & 0,2 & 0,14 & 0,39 \\
\hline \multirow{2}{*}{ Taq-1 } & \multirow{2}{*}{ rs731236 } & A & 306,5 & 0,76 & 0,72 & 0,71 & 0,74 & 0,93 & 0,6 & 0,69 \\
\hline & & G & 98,5 & 0,24 & 0,28 & 0,29 & 0,26 & 0,07 & 0,4 & 0,31 \\
\hline \multirow{2}{*}{ VPS13C } & \multirow{2}{*}{ rs8028149 } & $\mathrm{C}$ & 166 & 0,43 & 0,65 & 0,44 & 0,77 & 0,84 & 0,67 & 0,44 \\
\hline & & $\mathrm{T}$ & 224 & 0,57 & 0,35 & 0,56 & 0,23 & 0,16 & 0,33 & 0,56 \\
\hline \multirow{2}{*}{ SH3TC2 } & \multirow{2}{*}{ rs930205 } & C & 170 & 0,41 & 0,51 & 0,37 & 0,49 & 0,65 & 0,58 & 0,39 \\
\hline & & $\mathrm{T}$ & 242 & 0,59 & 0,49 & 0,63 & 0,51 & 0,35 & 0,42 & 0,61 \\
\hline \multirow{2}{*}{ SORBS2 } & \multirow{2}{*}{ rs955263 } & A & 187 & 0,45 & 0,42 & 0,43 & 0,47 & 0,5 & 0,23 & 0,41 \\
\hline & & $\mathrm{G}$ & 226 & 0,55 & 0,58 & 0,57 & 0,53 & 0,5 & 0,77 & 0,59 \\
\hline \multirow{2}{*}{$A B C A B$} & \multirow{2}{*}{ rs9893385 } & $\mathrm{A}$ & 231 & 0,56 & 0,52 & 0,53 & 0,57 & 0,51 & 0,41 & 0,58 \\
\hline & & G & 183 & 0,44 & 0,48 & 0,47 & 0,43 & 0,49 & 0,59 & 0,42 \\
\hline
\end{tabular}

\section{2.4. Data Analysis}

193 Epidemiological Data for this study was double entered using Microsoft ${ }^{\circledR}$ Access and validated to remove duplicates. To analyze the

194 population structure, we computed principal component (PC) using PLINK version 1.9. Ten (10) principal components were

195 computed using PLINK1.9's --pca command and the top two principal components (PC1 vs PC2) were plotted using R. A generalized

196 linear model (g/m) was also performed using the R statistical package to evaluate correlation between the PCs. The quality of the

197 variants was assessed, and association analysis was run with 28 high-quality variants using PLINK version 1.9 for the controls 
198 against all the cases put together. A total of 333 participants (113 cases; 181 controls), of which 161 were males and 172 were 199 females were included in the association analysis. What was the P values for statistical significance?

200

201

202

203

204

205

206

207

208

209

210

211

212

213

214

215

216

217

218 


\section{$219 \quad$ 3.0.Results}

\section{$220 \quad$ 3.1.Characteristics of studied cohorts}

221 The study population consisted of 793 individuals (323 TB individuals and 470 controls). The male gender was associated with active

222 TB in univariate logistics regression (odds ratio (OR) 1.78, 95\% confidence interval (CI). 1.23-2.57, $\mathrm{p}=0.002$ ). Young patients (less 223 than 35 years; $n=158)$ and patients older than 65 years $(n=11)$, were significantly associated with active TB $(p=0.001)($ Table 2$)$. 224 Analysis of risk factors revealed that $34 \%, 11.6 \%$ and $5.8 \%$ of the patients were registered as alcohol abusers, cigarette users and 225 substances abusers respectively

226 Information on the TB isolates ( $\mathrm{n}=323$ ) was available for 300 patients. The vast majority had pulmonary TB(97.0\%) followed by 227 Pulmonary TB with anemia (2.0\%), TB with lobber pneumonia (1.0\%) (Table 2). At the time of sampling, 10.0\% ( $\mathrm{n}=32$ ) of the 228 patients were hospitalized and $6.5 \%$ of the patients $(n=21)$ had previous history of TB. Analysis of risk factors revealed that $7.1 \%$ of 229 the patients were registered TB-Diabetes patients. Moreover, HIV-positive patients accounted for $5.8 \%$ of the study population and $2309.4 \%$ were notified as suffering from malnutrition. Sputum bacillary load at presentation was significantly higher in the MTBss group $231 \mathrm{n}=90$ patients having sputum grades of $2+$ compared to $(n=28)$ patients in the MAF group, $p<0.001$.). 
236 Table 2: Demographic characteristics of patients

\begin{tabular}{|c|c|c|c|c|c|}
\hline Characteristics & (TB patients) $\mathrm{N}=323$ & (NTB) $\mathrm{N}=469)$ & $\mathrm{P}$-value & OR & $95 \% \mathrm{CI}$ \\
\hline \multicolumn{6}{|l|}{ Gender } \\
\hline Male & 253 & 124 & $<0.001$ & 10.05 & $7.19-14.06$ \\
\hline Female & 70 & 345 & & Ref & \\
\hline \multicolumn{6}{|l|}{ Age } \\
\hline $13-24$ & 67 & 56 & $<0.001$ & 2.44 & $1.51-3.94$ \\
\hline $25-34$ & 91 & 78 & $<0.001$ & 2.37 & $1.53-3.70$ \\
\hline $35-44$ & 75 & 83 & 0.008 & 1.84 & $1.17-2.88$ \\
\hline $45-54$ & 55 & 112 & & Ref & \\
\hline $55-64$ & 24 & 66 & 0.300 & 0.74 & $0.42-1.31$ \\
\hline $65+$ & 11 & 74 & 0.001 & 0.30 & $0.15-0.62$ \\
\hline \multicolumn{6}{|l|}{ Risk factors } \\
\hline \multicolumn{6}{|l|}{$\mathrm{BCG}$} \\
\hline Yes & 200 & 313 & 0.163 & 0.81 & $0.60-1.09$ \\
\hline No & 123 & 156 & & Ref & \\
\hline \multicolumn{6}{|l|}{$\begin{array}{c}\text { Substance abuse (Cigarate } \\
\text { alcohol etc) }\end{array}$} \\
\hline Yes & 143 & 144 & $<0.001$ & 1.79 & $1.33-2.41$ \\
\hline No & 180 & 325 & & Ref & \\
\hline \multicolumn{6}{|l|}{ Diabetes } \\
\hline Yes & 20 & 3 & $<0.001$ & 10.25 & $3.02-34.80$ \\
\hline No & 303 & 466 & & Ref & \\
\hline \multicolumn{6}{|l|}{ HIV } \\
\hline Yes & 19 & 0 & & NA & \\
\hline $\mathrm{No}$ & 304 & 469 & & & \\
\hline \multicolumn{6}{|l|}{ Education } \\
\hline Primary & 51 & 132 & $<0.001$ & 0.47 & $0.32-0.69$ \\
\hline Secondary & 185 & 227 & & Ref & \\
\hline Tertiary & 42 & 15 & $<0.001$ & 3.43 & $1.85-6.39$ \\
\hline None & 45 & 95 & 0.009 & 0.58 & $0.39-0.87$ \\
\hline
\end{tabular}




\section{7}

\begin{tabular}{|r|c|c|c|c|c|}
\hline & & & & & \\
\hline Ethnicity & 125 & 113 & & Ref & \\
\hline Ekan & 47 & 71 & 0.025 & 0.60 & $0.38-0.93$ \\
\hline Ga & 95 & 264 & $<0.001$ & 0.32 & $0.23-0.46$ \\
\hline others & 56 & 21 & 0.002 & 2.4 & $1.37-4.23$ \\
\hline
\end{tabular}

238

239

240

241

242

243

244

245

246

247 


\section{$248 \quad$ 3.2.Identified MTBC Phylogenetic Lineages}

249 The 323 TB isolates after spoligotyping (S1) were identified as 79\% ( $n=255)$ MTBss and 21.1\% ( $n=68)$ MAF. Stratifying by Lineage,

$25087 \%(n=222 / 255)$ of the MTBss strains belonged to the Euro-American lineage, with sublineages Cameroon (50\%), Ghana (10.0\%)

251 and Haarlem (5.0\%) being the most prevalent and among Maf Lineage 5 (west African 1) (56\%) ( $n=38 / 68$. We detected a total of 66 252 spoligotypes, $286 / 323$ isolates (88.2\%) had previously defined shared type number (SIT). The remaining 37 isolates could not be 253 defined by the SITVIT database and thus were defined as 'orphan' [S1]

254

\subsection{Host genotype population structure and its association with Pathogen Lineages}

256 The SNPs investigated in our current study had similar allele frequencies compared to the global and African populations (Table 1).

257 The principal component analysis (PCA) plots coloured by case-control status, and by ethnicity show that the samples are 258 homogeneous based on the typed SNPs (Figure 1 and 2). Furthermore, the generalized linear model (gIm) showed no significant 259 correlation between the PCs and the case-control phenotype (Table 3). However, a glm for case-control status against age, weight, 260 and height revealed that the variables were significantly correlated with the phenotype based on the typed SNPs (Table 5) and were 261 thus included as covariates in the analysis [S2]. 


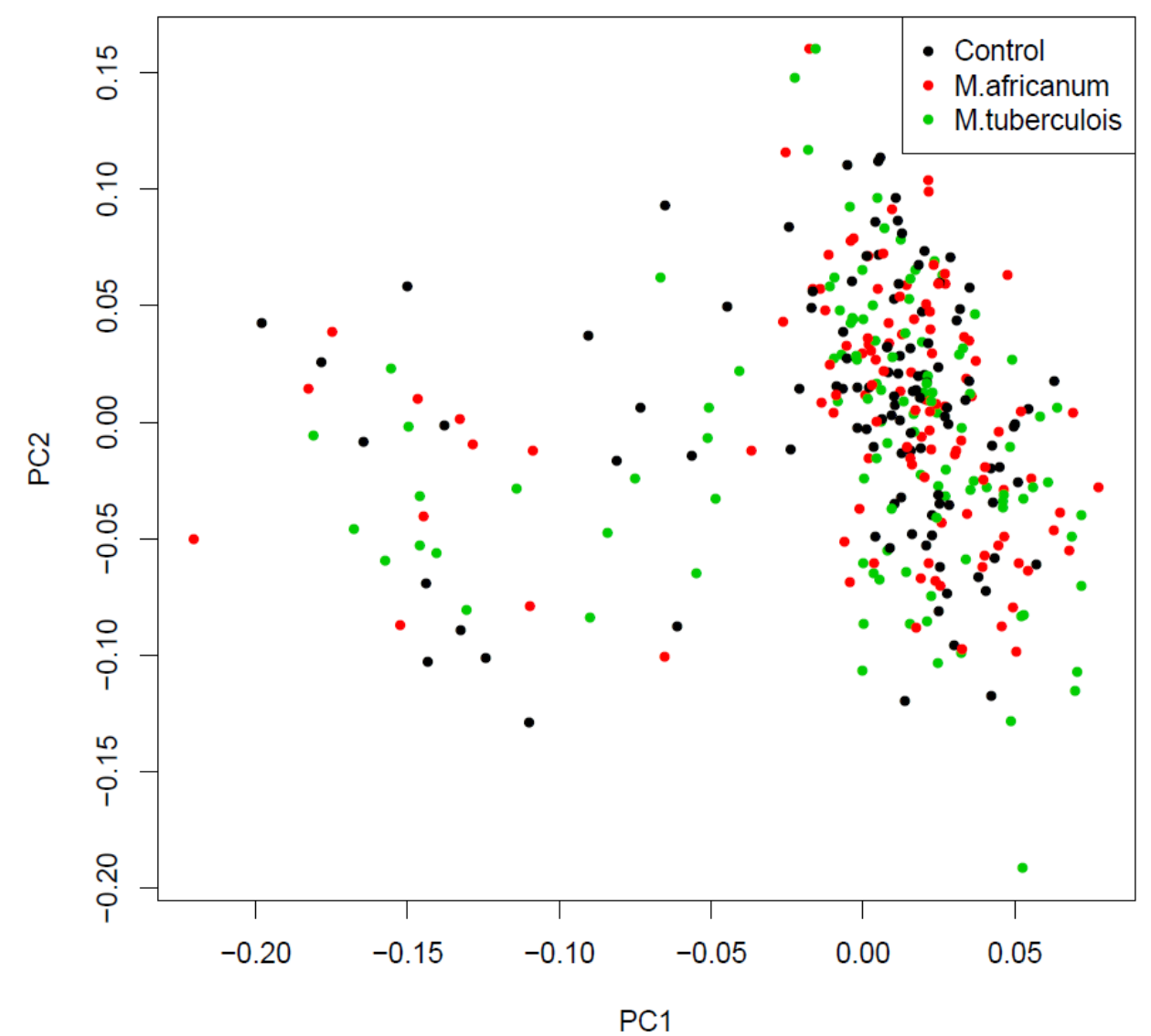

262

263 Figure 1: Principal component association plot between MTB cases and control 


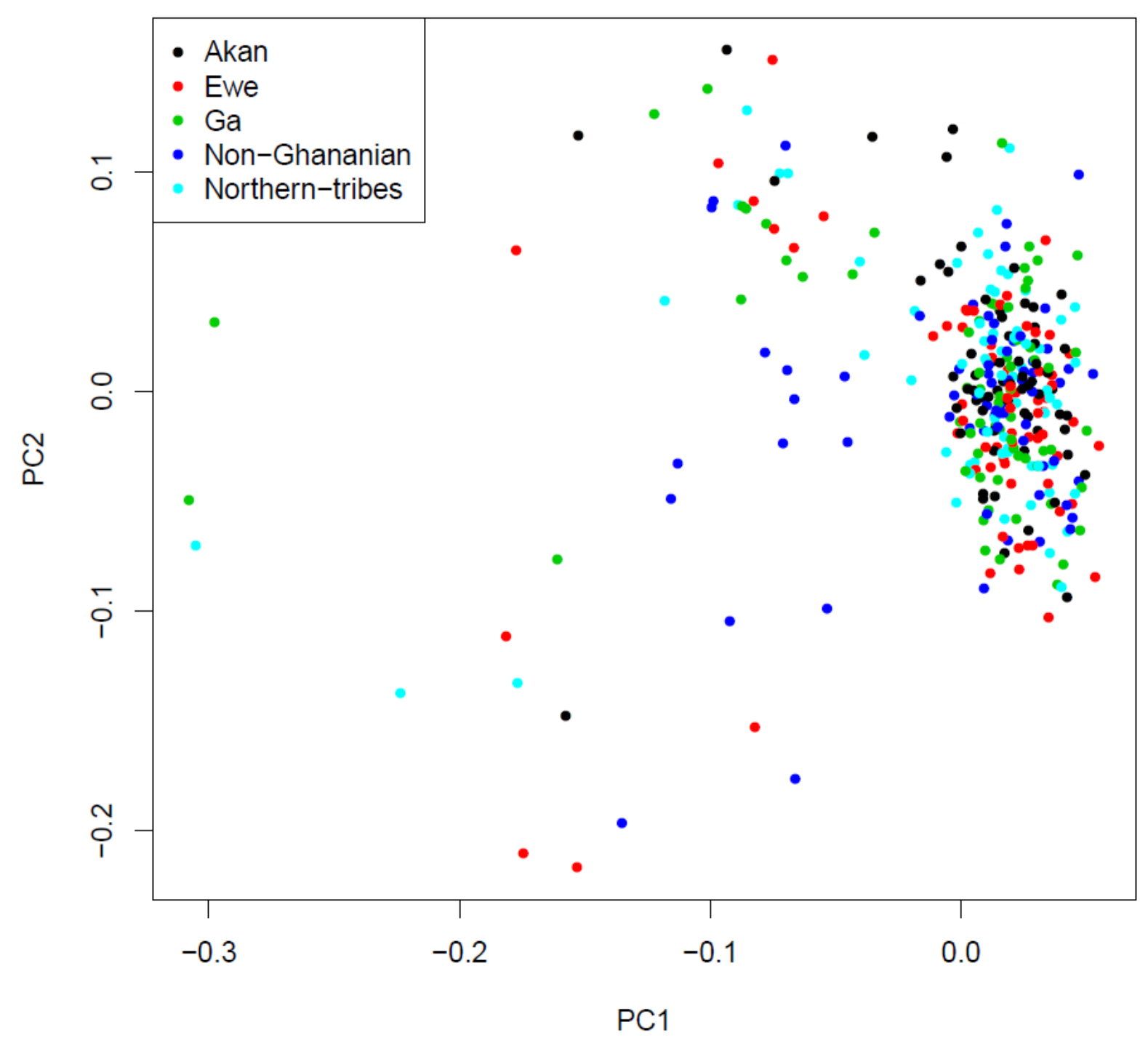

265 Figure 2: Principal component association plot between MTBC strains and patient ethnicity 
267 The variables that were correlated with the case-control phenotypes as earlier mentioned were included as covariates analysing the 268 association between the controls and all cases (MTBss and MAF). Two variants (rs17048476 and rs1482868) emerged after 269 adjusting for covariates. The variant rs17048476 is an intron variant on the AK124857 gene that has been previously found to be 270 associated with resistance to TB in highly susceptible individuals (Sobota et al 271 2016:https://www.ncbi.nlm.nih.gov/pubmed/26942285) (18). However, in our work, the rs17048476 (P=0.088, OR = 1.57, 95\% Cl = $2720.93-2.63)$ and $\operatorname{rs} 1482868(P=0.095, \mathrm{OR}=0.60,95 \% \mathrm{Cl}=0.33-1.09)$ were observed to be only suggestive compare to the high 273 significance observed by Sobota et al in their study (https://www.ncbi.nlm.nih.gov/pubmed/26942285) (Table 3). 


\section{Table 3: Association analysis of ccontrols vs all cases (MTBss + MAF)}

\begin{tabular}{|c|c|c|c|c|c|c|c|c|c|c|c|c|c|}
\hline CHR & POS & ID & REF & ALT & A1 & TEST & OBS_CT & OR & LOG(OR)_SE & L95 & U95 & Z_STAT & $\mathbf{P}$ \\
\hline 1 & 22206613 & rs2807348 & A & G & G & ADD & 333 & 1,08 & 0,29 & 0,61 & 1,90 & 0,25 & 0,8022 \\
\hline 1 & 57537459 & rs1524713 & C & $\mathrm{T}$ & $\mathrm{T}$ & ADD & 333 & 1,04 & 0,26 & 0,62 & 1,73 & 0,14 & 0,8865 \\
\hline 2 & 218389899 & rs2695342 & G & A & A & ADD & 333 & 2,13 & 0,78 & 0,46 & 9,77 & 0,97 & 0,3310 \\
\hline 2 & 218395009 & rs17235409 & G & A & $A$ & ADD & 333 & 0,95 & 0,44 & 0,40 & 2,25 & $-0,12$ & 0,9064 \\
\hline 2 & 218395009 & rs17235409 & G & $A$ & $A$ & ADD & 332 & 1,26 & 0,57 & 0,41 & 3,85 & 0,40 & 0,6870 \\
\hline 3 & 7987199 & rs17048476 & G & A & $A$ & ADD & 331 & 1,57 & 0,26 & 0,93 & 2,63 & 1,70 & 0,0883 \\
\hline 3 & 103407750 & rs12636260 & G & $T$ & $\mathrm{~T}$ & ADD & 326 & 0,78 & 0,29 & 0,44 & 1,36 & $-0,88$ & 0,3775 \\
\hline 4 & 61984804 & rs4860106 & A & G & G & $A D D$ & 329 & 1,30 & 0,26 & 0,79 & 2,15 & 1,03 & 0,3033 \\
\hline 4 & 185697150 & rs955263 & G & A & $A$ & $A D D$ & 328 & 0,74 & 0,26 & 0,44 & 1,24 & $-1,14$ & 0,2528 \\
\hline 5 & 113228247 & rs557438 & G & A & $A$ & $A D D$ & 321 & 0,76 & 0,28 & 0,44 & 1,31 & $-0,99$ & 0,3215 \\
\hline 5 & 148985671 & rs930205 & $T$ & C & $C$ & $A D D$ & 331 & 1,02 & 0,24 & 0,63 & 1,65 & 0,08 & 0,9359 \\
\hline 5 & 159263943 & rs4921437 & C & $\mathrm{T}$ & $\mathrm{T}$ & $A D D$ & 331 & 1,11 & 0,26 & 0,67 & 1,85 & 0,42 & 0,6729 \\
\hline 6 & 6202501 & rs1482868 & C & $T$ & $T$ & $A D D$ & 319 & 0,60 & 0,31 & 0,33 & 1,09 & $-1,67$ & 0,0958 \\
\hline 7 & 11609312 & rs2681052 & C & A & $A$ & $A D D$ & 331 & 1,22 & 0,25 & 0,75 & 1,99 & 0,80 & 0,4238 \\
\hline 7 & 72039275 & rs844669 & A & C & $C$ & ADD & 333 & 1,01 & 0,29 & 0,56 & 1,80 & 0,02 & 0,9806 \\
\hline 8 & 41260502 & rs4236914 & C & $\mathrm{T}$ & $\mathrm{T}$ & $A D D$ & 315 & 0,98 & 0,26 & 0,59 & 1,64 & $-0,06$ & 0,9492 \\
\hline 9 & 113674597 & rs3860173 & C & $T$ & $T$ & $A D D$ & 330 & 1,11 & 0,25 & 0,68 & 1,82 & 0,42 & 0,6745 \\
\hline 10 & 52771466 & rs 18004511 & C & $T$ & $\mathrm{~T}$ & $A D D$ & 333 & 1,02 & 0,26 & 0,61 & 1,72 & 0,08 & 0,9347 \\
\hline 10 & 52771482 & rs5030737 & G & A & $A$ & $A D D$ & 333 & 0,66 & 0,38 & 0,31 & 1,39 & $-1,10$ & 0,2722 \\
\hline 10 & 70761399 & rs2587469 & A & G & G & $A D D$ & 319 & 1,11 & 0,27 & 0,65 & 1,89 & 0,39 & 0,6986 \\
\hline 12 & 13855572 & rs7297313 & A & C & $C$ & $A D D$ & 312 & 1,10 & 0,24 & 0,68 & 1,76 & 0,39 & 0,6995 \\
\hline 12 & 46843681 & rs4768760 & A & C & $C$ & $A D D$ & 330 & 1,19 & 0,26 & 0,71 & 1,97 & 0,66 & 0,5124 \\
\hline 12 & 47844974 & rs731236 & A & G & G & ADD & 327 & 0,81 & 0,29 & 0,46 & 1,43 & $-0,72$ & 0,4708 \\
\hline 12 & 47846052 & rs1544410 & C & $T$ & $T$ & ADD & 332 & 1,20 & 0,29 & 0,68 & 2,11 & 0,64 & 0,5201 \\
\hline 14 & 98046406 & rs857063 & A & C & $C$ & ADD & 333 & 0,71 & 0,24 & 0,45 & 1,12 & $-1,46$ & 0,1437 \\
\hline 15 & 61724906 & rs8028149 & $T$ & C & $C$ & ADD & 320 & 1,26 & 0,27 & 0,74 & 2,14 & 0,86 & 0,3898 \\
\hline 16 & 7445185 & rs2346943 & $T$ & G & G & $A D D$ & 332 & 0,96 & 0,31 & 0,53 & 1,75 & $-0,12$ & 0,9006 \\
\hline 17 & 68822194 & rs9893385 & A & G & G & $A D D$ & 331 & 1,06 & 0,25 & 0,66 & 1,71 & 0,24 & 0,8108 \\
\hline
\end{tabular}

276 
277 The association analysis between the controls and all MTB cases identified the rs1482868 variant with a suggestive association to MTBss

$278(P=0.0681)$ This suggestive association was however absent when the two species were analyzed together (Table 4). It appears that possession

279 of this variants confers protection to being infected with MTBss (OR 0.55)

\section{Table 4: Association analysis of controls vs all MTB cases}

\begin{tabular}{|c|c|c|c|c|c|c|c|c|c|c|c|c|c|}
\hline CHROM & POS & ID & REF & ALT & A1 & TEST & OBS_CT & OR & LOG(OR)_SE & L95 & U95 & Z_STAT & $\mathbf{P}$ \\
\hline 1 & 22206613 & rs2807348 & A & G & $\mathrm{G}$ & $A D D$ & 294 & 1,16 & 0,31 & 0,64 & 2,12 & 0,48 & 0,6278 \\
\hline 1 & 57537459 & rs1524713 & C & $\mathrm{T}$ & $T$ & ADD & 294 & 1,02 & 0,28 & 0,59 & 1,77 & 0,07 & 0,9452 \\
\hline 2 & 218395009 & rs17235409 & G & A & A & ADD & 294 & 0,93 & 0,48 & 0,36 & 2,38 & $-0,16$ & 0,8753 \\
\hline 2 & 218395009 & rs17235409 & G & A & $A$ & $A D D$ & 293 & 1,53 & 0,58 & 0,49 & 4,76 & 0,73 & 0,4668 \\
\hline 4 & 61984804 & rs4860106 & A & G & $\mathrm{G}$ & ADD & 290 & 1,28 & 0,27 & 0,75 & 2,18 & 0,91 & 0,3648 \\
\hline 4 & 185697150 & rs955263 & G & A & A & ADD & 290 & 0,73 & 0,27 & 0,43 & 1,25 & $-1,13$ & 0,2565 \\
\hline 5 & 113228247 & rs557438 & G & A & $A$ & ADD & 284 & 0,75 & 0,30 & 0,41 & 1,35 & $-0,97$ & 0,3314 \\
\hline 5 & 148985671 & rs930205 & $\mathrm{T}$ & C & C & ADD & 292 & 0,94 & 0,26 & 0,56 & 1,56 & $-0,25$ & 0,8010 \\
\hline 7 & 72039275 & rs844669 & A & C & $C$ & ADD & 294 & 0,96 & 0,32 & 0,52 & 1,79 & $-0,13$ & 0,9005 \\
\hline 8 & 41260502 & rs 4236914 & C & $\mathrm{T}$ & $T$ & ADD & 276 & 1,05 & 0,28 & 0,60 & 1,81 & 0,16 & 0,8716 \\
\hline 9 & 113674597 & rs3860173 & C & $\mathrm{T}$ & $T$ & ADD & 291 & 1,02 & 0,27 & 0,60 & 1,72 & 0,06 & 0,9535 \\
\hline 10 & 52771466 & rs18004511 & C & $\mathrm{T}$ & $T$ & $A D D$ & 294 & 1,02 & 0,29 & 0,57 & 1,80 & 0,06 & 0,9554 \\
\hline 10 & 52771482 & rs5030737 & G & A & $A$ & $A D D$ & 294 & 0,61 & 0,42 & 0,27 & 1,39 & $-1,17$ & 0,2425 \\
\hline 10 & 70761399 & rs2587469 & A & G & $\mathrm{G}$ & ADD & 281 & 0,94 & 0,29 & 0,53 & 1,64 & $-0,23$ & 0,8192 \\
\hline 12 & 13855572 & rs7297313 & A & C & $C$ & $A D D$ & 275 & 1,05 & 0,26 & 0,63 & 1,74 & 0,18 & 0,8572 \\
\hline 12 & 46843681 & rs4768760 & A & C & C & ADD & 292 & 1,22 & 0,28 & 0,70 & 2,12 & 0,70 & 0,4854 \\
\hline 12 & 47844974 & rs731236 & A & G & $\mathrm{G}$ & ADD & 288 & 0,83 & 0,31 & 0,45 & 1,52 & $-0,61$ & 0,5405 \\
\hline
\end{tabular}




\begin{tabular}{|l|l|l|l|l|l|l|l|l|l|l|l|l|l|}
\hline $\mathbf{1 5}$ & $\mathbf{6 1 7 2 4 9 0 6}$ & rs8028149 & T & C & C & ADD & 283 & 1,31 & 0,29 & 0,75 & 2,30 & 0,94 & 0,3464 \\
\hline $\mathbf{1 6}$ & $\mathbf{7 4 4 5 1 8 5}$ & rs2346943 & T & G & G & ADD & 293 & 1,04 & 0,33 & 0,55 & 1,96 & 0,11 & 0,9141 \\
\hline $\mathbf{1 7}$ & $\mathbf{6 8 8 2 2 1 9 4}$ & rs9893385 & A & G & G & ADD & 293 & 1,03 & 0,26 & 0,62 & 1,73 & 0,12 & 0,9053 \\
\hline
\end{tabular}

283 The association analysis between the controls and $M$. africanum cases (MAF) showed that the rs2695342 variant among those 284 infected with MAF appeared to have the propensity to confer susceptibility to MAF infections $((P=0.093, \mathrm{OR}=8.35,95 \% \mathrm{Cl}=0.70-$ $28599.24)$ in this population.). This is different from the previous two variants with marginal significance. rs2695342 is a synonymous

$287 \quad 218390399 ; \mathrm{v}=\mathrm{rs} 2695342 ; \mathrm{vdb}=\mathrm{variation} ; \mathrm{vf}=55370148$ ) on the SLC11A1 gene and it has been previously associated with cutaneous leishmaniasis.

288

289

290

291

292

293

294

295

296

297 
300 Table 5: Association analysis of controls vs all MAF cases

\begin{tabular}{|c|c|c|c|c|c|c|c|c|c|c|c|c|c|}
\hline CHR & POS & ID & REF & ALT & A1 & TEST & OBS_CT & OR & LOG(OR)_SE & L95 & U95 & Z_STAT & $\mathbf{P}$ \\
\hline 1 & 22206613 & rs2807348 & A & G & $\mathrm{G}$ & $A D D$ & 220 & 0,71 & 0,49 & 0,27 & 1,84 & $-0,71$ & 0,4790 \\
\hline 1 & 57537459 & rs1524713 & C & $T$ & $T$ & $A D D$ & 220 & 1,04 & 0,38 & 0,50 & 2,19 & 0,11 & 0,9136 \\
\hline $2^{x}$ & 218389899 & rs2695342 & G & A & A & ADD & 220 & 8,35 & 1,26 & 0,70 & 99,24 & 1,68 & 0,0927 \\
\hline 2 & 218395009 & rs17235409 & G & A & A & $A D D$ & 220 & 0,56 & 0,58 & 0,18 & 1,74 & $-1,00$ & 0,3168 \\
\hline 2 & 218395009 & rs17235409 & G & A & $A$ & $A D D$ & 220 & 0,38 & 1,24 & 0,03 & 4,32 & $-0,78$ & 0,4368 \\
\hline 3 & 7987199 & rs17048476 & G & A & A & ADD & 219 & 1,48 & 0,39 & 0,69 & 3,18 & 1,01 & 0,3127 \\
\hline 3 & 103407750 & rs 12636260 & G & $T$ & $T$ & $A D D$ & 215 & 1,90 & 0,47 & 0,76 & 4,77 & 1,37 & 0,1695 \\
\hline 4 & 61984804 & rs4860106 & A & G & G & $A D D$ & 217 & 1,09 & 0,38 & 0,52 & 2,29 & 0,24 & 0,8127 \\
\hline 4 & 185697150 & rs955263 & $\mathrm{G}$ & A & A & $A D D$ & 216 & 0,73 & 0,40 & 0,33 & 1,59 & $-0,80$ & 0,4242 \\
\hline 5 & 113228247 & rs557438 & G & A & A & $A D D$ & 214 & 0,60 & 0,44 & 0,25 & 1,43 & $-1,15$ & 0,2493 \\
\hline 5 & 148985671 & rs930205 & $T$ & C & $C$ & $A D D$ & 218 & 1,17 & 0,38 & 0,56 & 2,46 & 0,43 & 0,6691 \\
\hline 5 & 159263943 & rs4921437 & C & $\mathrm{T}$ & $T$ & $A D D$ & 218 & 1,95 & 0,42 & 0,85 & 4,48 & 1,57 & 0,1153 \\
\hline 6 & 6202501 & rs1482868 & C & T & $T$ & $A D D$ & 214 & 0,77 & 0,47 & 0,31 & 1,93 & $-0,55$ & 0,5819 \\
\hline 7 & 11609312 & rs2681052 & c & A & $A$ & $A D D$ & 218 & 0,93 & 0,36 & 0,46 & 1,90 & $-0,20$ & 0,8451 \\
\hline 7 & 72039275 & rs844669 & A & C & $C$ & $A D D$ & 220 & 1,06 & 0,45 & 0,43 & 2,58 & 0,12 & 0,9025 \\
\hline 8 & 41260502 & rs4236914 & C & $\mathrm{T}$ & $T$ & $A D D$ & 210 & 0,86 & 0,43 & 0,37 & 1,98 & $-0,35$ & 0,7235 \\
\hline 9 & 113674597 & rs3860173 & c & $T$ & $T$ & $A D D$ & 218 & 1,48 & 0,39 & 0,68 & 3,20 & 0,99 & 0,3221 \\
\hline 10 & 52771466 & rs18004511 & C & $\mathrm{T}$ & $T$ & $A D D$ & 220 & 1,02 & 0,41 & 0,46 & 2,27 & 0,06 & 0,9527 \\
\hline 10 & 52771482 & rs5030737 & G & A & A & $A D D$ & 220 & 0,67 & 0,55 & 0,23 & 1,98 & $-0,72$ & 0,4688 \\
\hline 10 & 70761399 & rs2587469 & A & G & $\mathrm{G}$ & $A D D$ & 210 & 1,21 & 0,42 & 0,53 & 2,75 & 0,46 & 0,6468 \\
\hline 12 & 13855572 & rs7297313 & A & c & $C$ & $A D D$ & 204 & 1,84 & 0,39 & 0,85 & 3,97 & 1,56 & 0,1195 \\
\hline 12 & 46843681 & rs4768760 & A & c & C & ADD & 218 & 0,95 & 0,39 & 0,45 & 2,04 & $-0,12$ & 0,9052 \\
\hline 12 & 47844974 & rs731236 & A & G & $\mathrm{G}$ & $A D D$ & 215 & 0,85 & 0,43 & 0,37 & 2,00 & $-0,36$ & 0,7180 \\
\hline 12 & 47846052 & rs1544410 & C & $\mathrm{T}$ & $T$ & $A D D$ & 220 & 1,20 & 0,45 & 0,50 & 2,90 & 0,41 & 0,6807 \\
\hline 14 & 98046406 & rs857063 & A & C & C & $A D D$ & 220 & 0,64 & 0,35 & 0,32 & 1,28 & $-1,27$ & 0,2039 \\
\hline 15 & 61724906 & rs8028149 & $T$ & C & C & $A D D$ & 207 & 0,76 & 0,49 & 0,29 & 1,99 & $-0,55$ & 0,5808 \\
\hline 16 & 7445185 & rs2346943 & $T$ & G & $\mathrm{G}$ & $A D D$ & 220 & 0,99 & 0,49 & 0,38 & 2,60 & $-0,01$ & 0,9884 \\
\hline 17 & 68822194 & rs9893385 & A & G & G & $A D D$ & 219 & 1,02 & 0,41 & 0,46 & 2,27 & 0,06 & 0,9558 \\
\hline
\end{tabular}




\subsection{Discussion}

Host factors are increasingly being recognized as critical for TB control considering the diversity of the outcome of interaction between the MTBC and distinct human host populations. This study sought to explore potential host genetic factor (s) that may confer susceptibility or protection to distinct MTBC lineages, towards understanding crucial mechanisms of host-pathogen interaction. The main findings from this study were: 1) SNP rs2695342 on SIC11A1 gene has the propensity to confer susceptibility to MAF; 2) In addition, we also found that $7.1 \%$ of 393 adult TB patients studied had DM, three- old higher than the general population average of $2.0 \%$ and finally young patients less than 35 years and patients older than 65 years are associated with active TB in Ghana

In our study, rs2695342 (G/A) SIC11A1 gene had the propensity to confer susceptibility to TB caused by Maf. This variant is in introns of SIC11A1 gene (solute carrier family 11 member A1, previously known as natural resistance-associated macrophage protein 1 (Nramp1). This gene is a member of a family of metal ion-transport proteins whose cellular expression is restricted to phagocytic cells. SIC11A1 is a bivalent antiporter located on chromosome $2 q 35$ that delivers metal cations from the cytosol into acidic endosomal and lysosomal compartments where Fenon and Haber-Weiss reaction generates toxic antimicrobial radicals for direct antimicrobial activity against infectious microorganisms such as mycobacteria [19-21]. Basically, SLC11A1 may influence the survival of the TB pathogen after phagocytosis. We therefore suspect that the intronic position of this polymorphism might affect posttranscriptional modification of the affected gene hence potentially affecting the resulting SLC11A1 protein. Previous SLC11A1 studies in humans with TB in West Africans have primarily focused on four or five polymorphisms distributed across the gene: a $G T_{n}$ repeat in the $5^{\prime}$ promoter region, a four base-pair (TGTG) insertion/deletion (rs17235416) in the $3^{\prime}$ untranslated region (UTR), and two single nucleotide polymorphisms (SNPs) in intron 4 (rs3731865) and exon 15 (rs17235409, D543N). These mutations were found to be significantly associated with pulmonary TB. This association has been replicated in studies from Guinea-Conakry [21] and Gambia 
[21]. Our analysis shows that rs 2695342 might actually be a promotor/repression gene mutation which even though its synonymous could lead to significant phenotypic consequences. Mutations in this gene might eventually make the phagocytic cells less toxic thus making the patients more prone to infections by Maf.

Associations between particular MTBC lineages and human ethnicities have been observed before. Indeed, Lineage 1, 2 and 4 are reported to were strongly associated with Filipino, Chinese, and "white" ethnicities, respectively [22-23]. Likewise in China, Hui ethnicity was found to be associated with the Beijing family of MTBC [24]. Indeed, human genetic diversity has been linked to an increased or reduced susceptibility to TB. Recent studies have reported human genetic polymorphisms that influence the susceptibility to TB caused by Maf but MTBss or vice versa. In particular these studies indicate that human genetic susceptibility to TB is further influenced by the MTBC genotype. For example, a human polymorphism in 5-lipoxygenase (ALOX5) involved in the synthesis of leukotrienes and lipoxins and an important mediators of the inflammatory response has been associated with increased to TB risk caused by Maf [25]. Conversely, a human polymorphism reported recently in the Mannose Binding Lectin (MBL) was associated with protection against TB caused by Maf [26]. Moreover, this latter study also found that Maf bound human recombinant MBL more efficiently, perhaps leading to an improved uptake of Maf by macrophages and selection of deficient MBL variants among human populations exposed to Maf. Although our study did not find any significant association between ethnicity and MTBC lineages, our study suggests that host genetics play an important role in TB pathogenesis hence the need for newer approaches to TB therapy such as host directed immune-therapy, which have the potential to shorten the TB treatment and prevent resistance by promoting autophagy.

M. africanum is an important cause of human TB in West Africa, causing nearly $50 \%$ of all TB cases reported in West Africa. Our finding from this study that the MAF causes $21 \%$ of human TB in Ghana confirms our previous report from Ghana [27] and this finding is in agreement with agreement with existing findings in sub-Saharan Africa where MAF was previously found to cause $39 \%$ 
of human TB cases in Benin, Burkina Faso 18\%, Cameroon 56\%, The Gambia 39\%, Guinea Bissau 47\%, Ivory Coast 55\%, Nigeria $8 \%$, Senegal 20\%, Sierra Leone 24\%. Indeed, one potential reason for the stability of Maf in these countries is that the bacteria might have adapted to (some) human populations along the Gulf of Guinea. In addition, findings from our comparative genomics analysis of MAF from Ghana suggested potential adaptation of MAF L5 to a definitive host whereas MAF L6 exhibited traits of a pathogen with a wide host-range [28]. Therefore, the observation of MAF with these ethnic groups which mainly driven by L5 seems to suggest that L5 has indeed adapted to causing TB among the said ethnic groups. The Ga and the Ewe speaking ethnic groups traditionally form part of the Kwa people. The Kwa people of Africa includes the Ga-Adange, Ewe and Kwahu ethnic groups. Although the Ga, Ewe have different dialects of the same Kwa language family, members of theses individual groups are genetically interrelated]. Together they constitute the indigenous inhabitants of coastal West Africa.

Diabetes Mellitus (DM) is a known risk for tuberculosis (TB). Although our current report of $7.1 \%$ is lower than our previously reported figure of 9.4\% [29], the prevalence observed in this study is still three-times higher than in the general Ghanaian population prevalence of $2 \%$ [30]. In Sub-Saharan Africa, study findings regarding prevalence of diabetes mellitus amongst tuberculosis patients have been inconsistent. highly variable and differ by geographical region. The highest prevalence of diabetes mellitus among tuberculosis patients (38\%) (was reported in Nigeria [31], followed by Ethiopia 16\% [32], Tanzania (11\%) [33], Cameroon 9.5\% [33]. Because of the frequent co-morbidity of these two diseases, focusing on signs of diabetes among patients with TB, particularly if the risk factors are present, could contribute to improved detection and early treatment of diabetes in this population. Therefore, based on our findings, we recommend routine screening of TB patients for DM in addition to HIV which is the norm.

\subsection{CONCLUSION}


361 In conclusion, although our analyses do not include the previously studied SNPs, our findings implicate SLC11A1 as a potential

362 susceptibility gene of substantial interest for TB caused by MAF which is an important pathogen in West Africa

363 Supplementary figure 1: Sanger sequencing validation of TaqMan genotyping. Representative chromatogram for (A) the GG 364 genotype for rs5030737 (B) the GG genotype for rs17235409 (C) the GA genotype for rs17235409.

365

366

367

368

Conflict of interest statement: The authors declare no conflict of interest.

\section{Author contributions:}

\section{Funding:} DELTAS grant (107755/Z/15/Z: Awandare

\section{Acknowledgement:} during the study period

"Conceptualization, A.A.P, D.Y.M and A.W; methodology, A.A.P, P.M, P.A, K.M.H, S.Y.A, I.D.O, S.O.W, S.M.A, and K.M; formal analysis, A.A.P, P.A, K.M.H, I.D.O, S.M.A, and K.M; investigation, A.A.P, P.M, K.M.H, S.Y.A, S.M.A, and K.M; data curation, A.A.P, K.M.H, S,M,A, and K.M; writing-original draft preparation, A.A.P, D.Y.M and A.W.; writing-review and editing, A.A.P, P.M, P.A, K.M.H, S.Y.A, I.D.O, S.O.W, S.M.A, K.M., D.Y.M and A.W; project administration, A.A.P, D.Y.M and A.W.; funding acquisition, A.A.P. All authors have read and agreed to the published version of the manuscript

This study was funded by the World Bank African Centres of Excellence grant (ACE02-WACCBIP: Awandare); and Wellcome Trust

We express our gratitude to all laboratory staff and study participants of the various health facilities for their time and cooperation 


\section{$\underline{\text { References }}$}

1. Mediterr J Hematol Infect Dis 2013, 5(1): e2013070, DOI: 10.4084/MJHID.2013.070

2. Yeboah-Manu D, Asante-Poku A, Bodmer T, Stucki $D$, et al. Genotypic diversity and drug susceptibility patterns among $M$. tuberculosis complex isolates from South-Western Ghana. PLoS One. 2011; 6(7): e21906.

3. Gagneux S and Small PM. Molecular evolution of mycobacteria. in Handbook of tuberculosis, Vol. 1 (eds. Kaufmann, S.H. \& Rubin, E.) (Wiley VCH, Weinheim). 2008.

4. Malik AN, Godfrey-Faussett P. Effects of genetic variability of Mycobacterium tuberculosis strains on the presentation of disease. Lancet Infect Dis. 2005; 5(3): 174-83.

5. Sakamoto K. The pathology of Mycobacterium tuberculosis infection. Vet Pathol. 2012; 49(3): 423-39.

6. Narasimhan P, Wood J, MacIntyre CR, Mathai D, "Risk Factors for Tuberculosis," Pulmonary Medicine, vol. 2013;11.

7. Fattorini L, Piccaro G, Mustazzolu A, Giannoni F. Targeting Dormant Bacilli to Fight Tuberculosis. Mediterranean Journal of Hematology and Infectious Diseases. 2013; 5(1)

8. Pydi SS, Sunder SR, Venkatasubramanian S, Kovvali S, Jonnalagada S, et al. Killer cell immunoglobulin like receptor gene association with tuberculosis. Human Immunology 74 2013: 85-92

9. McHenry ML, Bartlett J, Igo RP, Jr, Wampande EM, Benchek P, Mayanja-Kizza H, et al. (Interaction between host genes and Mycobacterium tuberculosis lineage can affect tuberculosis severity: Evidence for coevolution? PLoS Genet. 2000: 16(4): e1008728

10. Comstock G. Tuberculosis in twins: a re-analysis of the Prophit Survey. Am Rev Respir Dis.1978;117:621-624

11. Mboowa G. Tuberculosis and Genetics of Sub-Saharan Africa Human Population. J Mycobac Dis. 2014;(4): 5

12. Asante-Poku A, Yeboah-Manu D, Otchere ID, Aboagye SY, Stucki D, Hattendorf J, et al. Mycobacterium africanum is associated with patient ethnicity in Ghana. PLoS Negl Trop Dis. 2015 8;9(1):e3370.

13. Government of Ghana (2010) Ghana Demographic and Health Survey, Final Report.

14. Zaidi SMA, Habib SS, Van Ginneken B, Ferrand RA, Creswell J, Khowaja S, Khan A. Evaluation of the diagnostic accuracy of Computer-Aided Detection of tuberculosis on Chest radiography among private sector patients in Pakistan. Sci Rep. 2018;8(1):12339.

15. Yeboah-manu D, Bodmer T, Owusu S, Ofori-adjei D, et al. Evaluation of Decontamination Methods and Growth Media for Primary Isolation of Mycobacterium ulcerans from Surgical Specimens. 2004: 5875-7

16. Yeboah-Manu D, Yates MD (2001) Stuart Mark (2001) Wilson Application of a simple multiplex polymerase chain reaction to aid in the routine work of the mycobacterium reference laboratory. J Clin Microbiol 2001:39: 4166-4168. 
410

411

412

413

414

415

416

417

418

419

420

421

422

423

424

425

426

427

428

429

430

431

432

433

434

435

436

437

438

439

440
17. Otchere, I. D. et al. Detection and characterization of drug-resistant conferring genes in Mycobacterium tuberculosis complex strains: A prospective study in two distant regions of. Tuberculosis (Edinb). 2016: 99, 147-154.

18. Kamerbeek J, Schouls L, Kolk A, van Agterveld M, van Soolingen D, Kuijper S, et al. Simultaneous detection and strain differentiation of Mycobacterium tuberculosisfor diagnosis and epidemiology. J Clin Microbiol. 1997: 35: 907-14.

19. Sobota RS, Stein CM, Kodaman N, Scheinfeldt LB, Maro I, Wieland-Alter W, et al. A Locus at $5 q 33.3$ Confers Resistance to Tuberculosis in Highly Susceptible Individuals. Am J Hum Genet. 2016:98(3):514-524.

20. Yakubu A., D. E. Alade and N. I. Dim: Molecular analysis of solute carrier family 11 member A1 (SLC11A1) gene in ruminants and non-ruminants using computational method- Genetika. 2014: 46, 925- 934

21. Cervino AC, Lakiss S, Sow O, Hill AV. Allelic association between the NRAMP1gene and susceptibility to tuberculosis in Guinea-Conakry. Ann Hum Genet. 2000;64:507-12

22. Kibirige D, Ssekitoleko R, Mutebi E, Worodria W. Overt diabetes mellitus among newly diagnosed Ugandan tuberculosis patients: a cross sectional study. BMC Infect Dis. 2013;13(1):12

23. Gagneux S, DeRiemer K, Van T, Kato-Maeda M, de Jong BC, et al. (2006) Variable host-pathogen compatibility in Mycobacterium tuberculosis. Proc Natl Acad Sci USA 103: 2869-287

24. Pang Y, Song Y, Xia H, Zhou Y, et al. (2012) Risk factors and clinical phenotypes of Beijing genotype strains in tuberculosis patients in China. BMC Infectious Disease. 2012; 12: 354

25. Herb F, Thye T, Niemann S, et al. ALOX5 variants associated with susceptibility to human pulmonary tuberculosis. Hum Mol Genet. 2008;17(7):1052-1060.

26. Thye T, Niemann S, Walter K, Homolka S, Intemann CD, et al. Variant G57E of Mannose Binding Lectin associated with protection against tuberculosis caused by Mycobacterium africanum but not by M. tuberculosis . 2011: 6: e20908

27. Asante-Poku A, Otchere ID, Osei-Wusu S, Sarpong E, Baddoo A, Forson A, et al. Molecular epidemiology of Mycobacterium africanum in Ghana. BMC Infect Dis. 2016 Aug 9;16:385.

28. Otchere ID, Coscollá M, Sánchez-Busó L, et al. Comparative genomics of Mycobacterium africanum Lineage 5 and Lineage 6 from Ghana suggests distinct ecological niches. Sci Rep. 2018;8(1):11269.

29. Asante-Poku A, Asare P, Baddoo NA, et al. TB-diabetes co-morbidity in Ghana: The importance of Mycobacterium africanum infection. PLoS One. 2019;14(2):e0211822. Published 2019 Feb 7. doi:10.1371/journal.pone.0211822

30. International Diabetes federation, 2020

31. Obiora NN, Azuonye OR, Oluoha NV, Uche OC. Prevalence of diabetes mellitus among pulmonary tuberculosis suspects in Nnewi, Nigeria. J Microbiol Biotechnol Res. 2017;6(2):1-5.

32. Damtew E, Ali I, Meressa D. Prevalence of diabetes mellitus among active pulmonary tuberculosis patients at St. Peter specialized hospital, Addis Ababa, Ethiopia. World J Med Sci. 2014;11:389-96 
441

442

443

444

445

446

447
33. Mugusi F, Swai A, Alberti K, McLarty D. Increased prevalence of diabetes mellitus in patients with pulmonary tuberculosis in Tanzania. Tubercle. 1990;71(4):271-6

34. Fonkeng LS, Ali IM, Noubom M, Bamou R, Sterve AH, Leo A, Kuiate J, Tume CB. Prevalence, Predictors and Treatment Outcome of Type 2 Diabetes among Newly Diagnosed Sputum Positive Pulmonary Tuberculosis Patients in Western Cameroon. J Infect Dis Epidemiol. 2017;3:2 